\title{
Sur une propriété de l'opération $A$.
}

Par

Otton Nikody m (Cracovie).

MM. Lusin et Sierpiński ont étudié une opération très générale sur une infinité dẻnombrable d'ensembles, dite opération $A^{1}{ }^{1}$, et définie comme il suit.

Supposons qu'à tout système fini de nombres naturels $n_{1}, n_{2}, \ldots, n_{k}$ corresponde un ensemble $E_{n_{1}, n_{2}, \ldots, \pi_{k}}$.

Désignons par $E$ l'ensemble de tous les éléments $x$, tels que pour chacun d'eux au moins une suite infinie d'indices $n_{1}, n_{3}, n_{3}, \ldots$ existe telle que $x$ appartienne à chacun d'ensembles

$$
E_{n_{1}}, E_{n_{1}, n_{n}} E_{n_{1}, n_{2}, m_{2}}, \ldots
$$

On dit que l'ensemble $E$ est le résultat d'une opération $A$, effectuée sur le système d'ensembles $S=\left\{E_{n_{1}, n_{2}, \ldots, n_{k}}\right\}$.

MM. Lusin et Sierpiński ont démontré dans lo mémoire cité que l'opération $A$, effectuée sur un système d'ensembles mesurables $(L)$, donne toujours un ensemble mesurable $(L)$. En modifiant convenablement la démonstration de ces auteurs, je prouverai la proposition suivante:

Théorème. L'opération A effectuée sur un système d'ensembles jouissants de la propriété, de Baire donne toujours un ensemble joutissant de la propriété de Baire.

Soit $P$ un ensemble parfait donné (dans l'espace à $m$ dimensions). Nous dirons qu'un ensemble $E$ est sur $P$ de première caté-

1) N. Lüin et W. Sierpiński: Sur quelques proprétés des ensembles (A), Bulletin de l'Acad. des Sciences de Cracovie 1918, p. 47 et 48. 
gorie au point $p$ (appartenant à $E$ ou non), s'il existe une sphère (m-dimensionnelle) $S$ entourant $p$ et telle que l'ensemble $P E S$ est de première catégorie sur $P$; s'il n'existe pas une telle sphère, nous dirons que $E$ et sur $P$ de deuxième catégorie au point $p^{1}$ ).

On dit qu'un ensemble $E$ est sur $P$ partout de deuxième catégorie, s'il est sur $P$ de deuxième catégorie en tout point $p$ de $P$. On démontre sans peine que si l'ensemble $E$ est sur l'ensemible parfait $P$ de deuxième catégorie, il existe une portion $I I$ de $P$, telle que $E$ est sur $\Pi$ partout de deuxième atégorie ${ }^{2}$ ).

On dit qu'un ensemble $E$ jouit de la propriété de Baire, s'il n'existe aucun ensemble parfait $P$, sur lequel $F$ et son complémentaire $C E$ seraient partout de deuxième catégorie. Nous dirons qu'un ensemble $E$ jouit de la propriété de Baire relativement à l'ensemble parfait $P$, s'il n'existe aucune portion $\Pi$ de $P$, sur laquelle $E$ et $C E$ seraient partout de deuxième catégorie.

En démontrant notre théorème nous nous bornerons au cas où $P$ est l'ensemble de tous les nombres réels. On verra sans peine quelles modifications il faudra faire pour le cas où $P$ est un ensemble parfait quelconque, situé dans l'espace à $m$ dimensions.

Au lieu de dire que l'ensemble $E$ jouit de la propriété de Baire relativement à l'ensemble de tous les nombres réels, nous dirons, pour abréger, que $E$ est un $\beta$. On voit sans peine que pour démontrer qu'un ensemble $E$ est un $\beta$, il suffit de prouver qu'il existe un ensemble $H \subset E$ qui est un $\beta$ et qui est en tout point $p$ de la même catégorie (sur l'ensemble de tous les nombres réel) que l'enemble $E$.

Lemme I. Si les ensembles $M$ et $Q \subset M$ sont des $\beta$, tous les deux de la même catégorie en tout point, l'ensemble $R=M-Q$ est de première catégorie.

Dém. Si l'ensemble $R$ était de deuxième catégorie, il serait partont de deuxième catégorie dans un intervalle $\delta$, et, à plus forte raison, l'ensemble $M \supset R$, donc aussi l'ensemble $Q$, serait partout de deuxième catégorie dans $\delta$. Les ensembles $R=M-Q$ et $C R \supset Q$ seraient done tous les deux partout de deuxième catégorie dans $\delta$,

1) X. Lusin et W. Sierpinski Journäl de Mathématiques 7-e série t. II. (1923), p. 68-69.

y) Voir p. e. H. Lebesgae: Journ. de Math., 6-e série t. I (1905) p, 185. 
ce qui est impossible, puisque $R$, comme différence de deux $\beta$. est un ensemble $\beta$. Notre lemme est ainsi démontré.

Lemme II. Si $H$ est un $\beta$ et $E C H$, il existe un ensemble $M$ qui est un $\beta$, de la même catégorie que $E$ en tout point, tel que $E \subset M \subset H$.

Dém. Soit $Q$ l'ensemble de tous les points $p$ (appartenant à $E$ ou non) en lesquels $E$ est de deuxième eatégorie. Posons $M=H Q+E$ : on voit sans peine que l'ensenble $M$ satisfait aux conditions de notre lemme.

En effet, l'ensemble de tous les points $p$ en lesquels $E$ est de $1^{\text {re }}$ catégorie est lui même de $1^{\text {re }}$ eatégorie, donc le complémentaire de $Q$. et, à plus forte raison', celui de $Q+E$, est de $1^{\text {re }}$ catégorie, d'où résulte-que l'ensemble $Q+E$ est un $\beta$. L'ensemble $M$, comme produit de deux $\beta$, est douc un $\beta$. Or, si $E$ est de $1^{\text {re }}$ catégorie an point $p$, il existe un intervalle $\Delta$ entourant $p$, tel que $E \Delta$ est de $1^{\text {ra }}$ catégorie, douc $\Delta Q=0$ et $M \Delta$ est de $1^{\text {re }}$ catégorie, ce qui prouve que $M$ est de $1^{\text {re }}$ catégorie au point $p$. D'autre part, si $E$ est de deuxième catégorie au point $p$, il en est de même, à plus forte raison, de l'ensemble $M \supset E$. Notre lemme est ainsi démontré.

Lemme III. Si $M_{n}(n=0,1,2, \ldots)$ est une suite. infinie d'ensembles $\beta, E_{n}-$ un ensemble $\subset M_{n}$ qui est en tout point de la même catégoric que $M_{n}($ pour $n=0,1,2, \ldots)$. et si $E_{0}=E_{1}+E_{2}+E_{\mathrm{s}}+\ldots$. l'ensemble $M_{0}-\left(M_{1}+M_{2}+\ldots\right)$ est de première catégorie.

Dém. Posons $T=M_{1}+M_{2}+M_{3}+\ldots, R=M_{0}-T, Q=$ $=M_{0}-R=M_{0} T$ : l'ensemble $Q$ sera done uu $\beta$ (comme produit de deux $\beta$ ). D'après $E_{0}=E_{1}+E_{\mathrm{a}}+\ldots$ et $E_{n} \subset M_{n}$ (pour $n=0$, $1,2, \ldots)$, nous aurons $E_{0} \subset M_{0}$ et $E_{0} \subset T$, donc $E_{0} \subset M_{0} T=Q$. Il est done $E_{0} \subset Q \subset M_{0}$ : les ensembles $Q$ et $M_{0}$ sout done en tout point dé la même calégorie; $Q$ et $M_{0}$ écant des $\beta$, il ei résulte, d'après le lemme I. que l'ensemble $R=M_{0}-Q$ est de première catégorie, e. q. Ł. d.

Soit maintenant $E$ un eusemble qui est le résultat de l'opération $A$ effectuéz sur le système d'ensembles $S=\left\{E_{n_{3}, n_{1} \ldots \ldots, n_{k}}\right\}$ et supposons que les ensembles $E_{u_{t} \cdot n_{i} \ldots \ldots n_{k}}$ sont tous des $\beta$. On voit sans peine 
qu'on peut supposer que le système $S$ est régulier, c'est-à-dire qu'on a toujours

$$
E_{n_{1}, n_{2}, \ldots, n_{k}} \supset E_{n_{1}, n_{2}, \ldots, n_{k}, n_{k+1},}
$$

puisque si le système $S$ ne satisferait pas à cette condition, il suffirait de remplacer tout ensemble $E_{n_{1}, n_{2}, \ldots, n_{k}}$ par le produit $E_{n_{1}} E_{n_{1}, \ldots, \ldots} E_{n_{1, n}, \ldots, n_{k}}$ et d'observer qu'un produit fini d'ensembles $\beta$ est un $\beta$.

Désignons généralement par $E^{p_{1}, p_{2} \ldots, p_{s}}$, l'ensemble déterminé par le système

où

$$
S^{p_{1}, n_{2}, \ldots, p_{t}}=\left\{E_{n_{1}, n_{2}, \ldots, n_{k}}^{p_{1}, p_{2}, \ldots, p_{k}}\right\}
$$

$$
E_{n_{1}, n_{1}, \ldots, n_{k}}^{r 1, n_{k}, \ldots, n_{k}}=E_{p_{1,}, p_{1}, \ldots, p_{k}, n_{1}, n_{2}, \ldots, n_{k}}, \text { pour } n_{1}, n_{2}, \ldots, n_{k}=1,2,3, \ldots
$$

Le système $S$ érant régulier, nous aurons évidemment:

$$
E^{p_{1}, p_{2}, \ldots, p_{1}} \subset E_{p_{1, p 2}, \ldots, p_{1}}
$$

Or, on voit sans peine que

$$
E=E^{1}+E^{2}+E^{3}+\ldots
$$

et

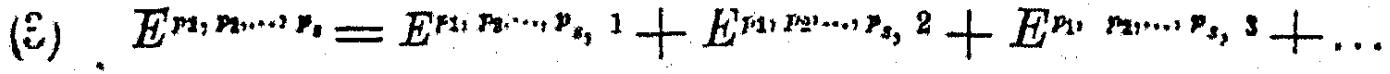

Les ensembles $E_{n_{1}, n_{n} \cdots, n_{k}}$ étant des $\beta$, il existe, d'après (1) et en vertu du lemme II, pour tout système d'indices $p_{1}, p_{2}, \ldots p$, un ensemble $M^{m}$ mi...p qui est un $\beta$, de la mêmè catégorie en tout point que l'ensemble $E^{p_{1}, p_{2}, \ldots, p_{2}}$ et tel que

$$
E^{p_{2}, p_{2}, \ldots, p_{s}} \subset M^{p_{1}, \ldots, p_{2}} \subset E_{p_{1}, p_{2}, \ldots, p_{s},}
$$

et il existe aussi un ensemble $M$ qui est un $\beta$, de la même catégorie en tout point que l'ensemble $E$, tel que

\section{Posons}

$$
E \subset M
$$

$$
T=M^{1}+M^{2}+M^{3}+. . .,
$$

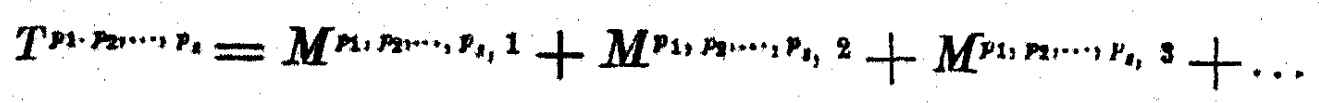

- ce seront des ensembles $\beta$, en tant que sommes d'infinités dénombrables d'ensembles $\beta$. Posons encore:

$$
\begin{aligned}
& R=M-T, \\
& R^{p_{1}, p_{1}, \ldots, p_{4}}=M^{p_{1}, p_{2}, \ldots, p_{d}}-T^{p_{1}, p_{3} \ldots, p_{p_{1}}},
\end{aligned}
$$




$$
N=R+\Sigma R^{p_{1} p_{3}, \cdots, p_{s}},
$$

la sommation $\Sigma$ s'étendant à tous les systèmes finis de nombres. naturels $p_{1}, p_{2}, \ldots, p_{*}$.

Les ensembles $M$ et $M^{p_{1} r_{2} \ldots, p_{s}}$ étant des $\beta$, nous concluons d'après (5), (6) et (8), resp. d'après (4), (7) et (9), et en vertu du lemme III, que les ensembles $R$ et $R^{p_{1}, p_{2}, \ldots, p_{p_{1}}}$ sont de première catégorie. Donc, d'après (10), l'ensemble $N$ est de $1^{\text {re }}$ catégorie (en tant que somme d'une infinité dénombrable d'ensemble de $1^{\text {re }}$ catégorie). L'ensemble

$$
H=M-N
$$

est done un $\beta$ (comme différence de deux $\beta$ ), de la même catégorie en tout point que l'ensemble $M$ (puisque $N$ est de $1^{\text {re }}$ catégorie), donc aussi que $E$. D'après la remarque faite plus haut, il suffira donc de prouver que $H \subset E$, pour démontrer que l'ensemble $E$ est un $\beta$.

Soit donc $x \varepsilon H$. D'après (11) nous avons donc $x \varepsilon M$ et $x$ non $\varepsilon N$, donc, d'après (10), $x$ non $\varepsilon R$, ce qui donne, d'après (8): $x \varepsilon T$. D'après (6) il existe donc un indice $n_{1}$, tel que $x \varepsilon M^{n_{1}}$. D'après (10) la formule $x$ non $\varepsilon N$ donne $x$ non $\varepsilon R^{n_{1}}$, donc, d'après $x \varepsilon M^{n_{1}}$ et d'après (9), $x \varepsilon T^{n_{1}}$. D'après (7) il existe done un indice $n_{2}$, tel que $x \varepsilon M^{n_{1} n_{3}}$ En repelant notre raisonsement, nous obtenons une suite infinie d'indices $n_{1}, n_{2}, n_{\mathrm{g}}, \ldots$, telle que

$$
x \in M^{n_{1}} . M^{n_{1} n_{2}} \cdot M^{n_{1}, n_{2}, n_{2}} \ldots .
$$

ce qui donne, d'après (4):

$$
x \in E_{n_{1}} E_{n_{1}, n_{2}} E_{n_{n_{1}}, n_{2}, x_{3}} \ldots
$$

et prouve que $x \varepsilon E$.

Nous avons ainsi prouvé que $H C E$. et nous pouvons regarder notre théorème comme démontré.

Tout ensemble $(A)$ de M. So uslin pouvant être regardé comme résultat de l'opération $A$ effectuée sur les intervalles (ou, plus généralement, sur les ensembles fermés), il résulte tout de suite de notre théorème le suivant

Corollaire: Tout ensemble (A) jouit de la propriété de Baire.

Cette propriété importante des ensembles $(A)$ a été démontré 
en 1923 sur une autre roie par MM. Lusin et Sierpinski 1 ). Vue l'existance des ensembles $(A)$ non mesurables $(B)$, il en résulte l'existence des ensembles non mesurables $(B)$; jouissant de la propriété do $\mathrm{Baire}$, ce qui resoût négativement un problème difficile posé par M..Lebesgue sur les fonctions représentables analytiquement ${ }^{2}$ ).

1) Journal de Mathérmatiquss, 7-9 sério t. II, p. 68 ss.

j) Journ. de Math. 6-e série, t. I, (1905), p. 188. 